Notre Dame Journal of Formal Logic

Volume 41, Number 3, 2000

\title{
FREGE'S NEW SCIENCE
}

\author{
ALDO ANTONELLI AND ROBERT MAY
}

\begin{abstract}
In this paper, we explore Fregean metatheory, what Frege called the New Science. The New Science arises in the context of Frege's debate with Hilbert over independence proofs in geometry and we begin by considering their dispute. We propose that Frege's critique rests on his view that language is a set of propositions, each immutably equipped with a truth value (as determined by the thought it expresses), so to Frege it was inconceivable that axioms could even be considered to be other than true. Because of his adherence to this view, Frege was precluded from the sort of metatheoretical considerations that were available to Hilbert; but from this, we shall argue, it does not follow that Frege was blocked from metatheory in toto. Indeed, Frege suggests in Die Grundlagen der Geometrie a metatheoretical method for establishing independence proofs in the context of the New Science. Frege had reservations about the method, however, primarily because of the apparent need to stipulate the logical terms, those terms that must be held invariant to obtain such proofs. We argue that Frege's skepticism on this score is not warranted, by showing that within the New Science a characterization of logical truth and logical constant can be obtained by a suitable adaptation of the permutation argument Frege employs in indicating how to prove independence. This establishes a foundation for Frege's metatheoretical method of which he himself was unsure, and allows us to obtain a clearer understanding of Frege's conception of logic, especially in relation to contemporary conceptions.
\end{abstract}

\section{Frege, Hilbert, and Metatheory}

A little over a century ago a correspondence commenced between one of the most well-known mathematicians of the day and a lightly regarded colleague in Jena. This correspondence, between Hilbert and Frege, while of some but apparently not great interest to Hilbert, was thought by Frege to broach issues of central importance, so much so that he suggested that it warranted publication. ${ }^{1}$ Since Hilbert was unresponsive to this suggestion, Frege proceeded to write up his side of the discussion in a pair of articles under the title Die Grundlagen der Geometrie [13] in 1903. In response to

Received March 29, 2001; printed September 30, 2002

2001 Mathematics Subject Classification: Primary, 03A05; Secondary, 00A30

Keywords: Frege, Hilbert, logicism, logical truth, logical constants, independence proofs (C) 2001 University of Notre Dame 
a critique of his views by Korselt [24], Frege published a more elaborated set of three articles under the same title in 1906 [14]. Taken together, these works represent the most detailed presentation of Frege's views between the publications of the second volume of Grundgesetze der Arithmetik [12] in 1903 and the series of three articles collectively known as Logical Investigations [16] that were published in the 1920s.

Reading over Frege's side of the discussion, it is apparent that the issue that animated Frege in these papers is the status of axioms. Frege felt that his view represented a traditional understanding of this notion, and that Hilbert's departure from this understanding led to a confusion about axioms that undermined many of the sorts of results, in particular, the independence of the axioms of geometry, that Hilbert saw as major mathematical achievements. Symptomatic of Hilbert's confusion, according to Frege, was Hilbert's claiming that axioms could serve to define; the reason that this is a confusion, according to Frege, is that axioms and definitions are statements of wholly different types. Axioms are a sort of contentful proposition, and as such they express thoughts; consequently, "an axiom must contain no unknown sign, for otherwise it would express no thought at all" ([14], p. 52-53). Axioms could not serve to define, for they contain nothing in need of definition: "To definitions that stipulate something, I opposed principles and theorems that assert something. The former contain a sign (word, expression) that is still to receive a reference by means of them; the latter contain no such sign." Frege saw Hilbert's confusion on this score as no small matter; it was rather a confusion at the very foundations of logic.

Of late, there has been much focus on the dispute between Frege and Hilbert, in large part because it has been thought to be particularly revealing of certain peculiar aspects of Frege's view of logic which, from today's perspective, may seem odd. The considerations that drove his critique of Hilbert, so it is argued, effectively prevented Frege from being able to adopt a metatheoretical stance toward logic, consequently blinding Frege to the sorts of results about logic that can be established once logic is distinguished from metalogic. As commentators on this issue have remarked, this is not merely a matter of the evolution of ideas; it is not just the banal observation that before there could be Tarski's insights, there had to be Frege's. Rather, the lack of metatheory in Frege is to be traced back to the assumption, at the heart of his view of logic, that logic is the universal system of reasoning; it is something that is reasoned in, not about. ${ }^{2}$ This is not to say, however, that Frege did not have metatheoretical insight; Frege often makes what we would consider metatheoretical remarks in the way of justifying aspects of logic. For example, he justifies rules of inference in terms of their soundness. ${ }^{3}$ What he does not do, however, is provide a proof of soundness and indeed, the argument goes, could not, for that would require metalogic.

It seems to us undoubtedly right that there is something very different about the way Frege viewed logic and that this difference lies at the heart of his view of logic. What does not seem right to us, however, is that this difference precluded Frege from metatheory. Frege himself apparently did not think so; in the third installment of the 1906 version of Die Grundlagen der Geometrie [14] he outlines a method for proof of the independence of propositions distinct from Hilbert's, giving a prima facie indication that he did not perceive any incompatibility per se between an approach to metatheory and fundamental assumptions about logic, including those which he faults Hilbert for failing to heed. Nevertheless, Frege recognized that he was entering 
unchartered logical territory, sufficiently unexplored so as to warrant caution; moreover, it is clear that Frege did not see obtaining such results as a central goal of logical inquiry and he never returned to the topic after 1906.

If nothing in Frege's conception of logic inherently precluded metatheory, there are nonetheless aspects of his approach that cast metatheory in a very different light than that in which we have come to know it today in its genesis from Hilbert and more proximately Tarski. To see this, however, we must understand how Frege departed in his view of semantics, in particular, the relation of language and interpretation, from certain assumptions that we find in Hilbert. As we shall discuss in the next section, it is over these assumptions that Frege took Hilbert to task. Because of his adherence to his particular presuppositions about this relation, and not to any notion of the universality of logic, Frege was indeed blocked from the sort of metatheoretical considerations that were available to Hilbert. But as we shall argue in Section 2, it does not follow from these considerations that Frege was blocked from metatheory tout court, although to the extent that metatheory is open to Frege, there will be a significant divergence in its scope and applicability as compared to the contemporary view, Frege's conception being much the more circumscribed. In Section 3 we will examine Fregean metatheory, the application of logic he called the "New Science" which he took to be concerned with establishing certain sorts of relations between propositions, the particular case of concern to Frege being their independence from one another. At this juncture, there is a certain commonality between Frege and Hilbert as to the enterprise; they were at odds, however, on how to undertake that enterprise, given the differences (outlined in Section 1) about the nature of language. There was an aspect of his New Science upon which Frege remarks, however, that led him to a certain reserve about it; this is the apparent need to stipulate the logical terms, those terms that must be held invariant to obtain proofs of independence. In Section 4 we will give reasons why skepticism on this is perhaps unwarranted, for we shall show that within the parameters Frege establishes for the New Science, a characterization of logical truth and logical constant can be obtained by a suitable adaptation of the permutation argument Frege employs in indicating how to prove independence. This result, which goes beyond what Frege himself envisaged, establishes a foundation for Frege's metatheoretical method that he himself was unsure of but which places us in a position to undertake a more coherent and measured evaluation of Frege's conception of logic, in particular, his view of the possibility of metalogic, as this separates him from much of modern logic.

\section{Interpreted and Uninterpreted Languages}

As Frege saw his dispute with Hilbert, it turned on the following, to Frege unassailable, claim:

(Ax) Axioms are true and could not be otherwise.

Frege's endorsement of (Ax) is meant to include the strong modality; what he meant was not just the truism that no false proposition could be an axiom, something that no one would dispute, least of all Hilbert, but rather that if a proposition is genuinely an axiom, then it is not even sensible to consider it to be other than true. This, as we shall see, is a consequence of the doctrine of sense and reference, so if it were possible to consider axioms to be other than true, they would be excluded from the domain of contentful propositions, that is, those that express thoughts that determine a truth 
value, an absurd result to Frege's mind. By way of contrast, for Hilbert, and indeed for modern logic generally, not holding the second clause is the gateway to metatheory. More precisely, the way is opened by the insight that axioms do not have fixed interpretations, implying that while axioms may be true under some interpretations, they may be false under others. Thus, that there can be non-Euclidean geometries, in which the axiom of parallels is false, but the other geometrical axioms are true, proves the independence of the former from the latter in Euclidian geometry. (In modern parlance, Hilbert proves independence of $P$ from $A_{1}, \ldots, A_{n}$ by giving a model of $\left\{A_{1}, \ldots, A_{n}, \neg P\right\}$.) To Frege, this makes sense only as a play on the word axiom that distorts its original and properly intended meaning; Frege repeatedly speaks of the notion of axiom, as he uses it, as "traditional" and "expedient"; in contrast, Hilbert's notion is seen as somehow deviant and leading to confusion. Needless to say, Hilbert for his part saw no such lack of clarity.

Looking at matters from Frege's side of the fence, two matters present themselves at this point. First, to what can we trace Frege's views about axioms; from what source of underlying assumptions does his stubborn attachment to the proposition above flow, in particular to its second clause, in distinction to Hilbert? Second, to what extent does his acceptance of the proposition's second clause deny Frege access to metatheory? If having metatheory requires dropping adherence to this clause, then, of course, denial is total. But as already noted, Frege did not see things this way; having some metatheory he thought was compatible with it. But what sort? In this section, it is to the former question that we will address ourselves; the latter will be postponed to Section 3.

So what is the answer to our first inquiry? To what prior views can we trace the roots of Frege's objections to Hilbert? Our answer is that they are to be found in a fundamental difference between Frege and Hilbert in what they understood a language (that is, a Begriffsschrift) to be. Consider first Frege's view.

For Frege, the basic linguistic notion is that of a sign, pairings of a symbol (a formal mark) and a sense, such that the symbol expresses the sense. A language is a system of signs, such that any change in the pairing of symbols and senses would be a change in the signs and would thus be a different system of signs, and hence a different language. ${ }^{5}$ Frege labels propositions as those concatenations of signs that express thoughts. Thus a language, a bit more precisely, can be characterized as a set of propositions. Reasoning is carried out in a language in this sense, and it will be facilitated by a properly designed language in which all the signs are pairwise disjoint from each other, since then any two distinct symbols - that part of the sign open to inspection-will also express different senses. The method of reasoning (that is, proof) is an ordered sequence of propositions; what a proof shows is how true thoughts follow from other true thoughts. Establishing that a sequence of propositions is a proof, however, is something that is determined strictly in virtue of the symbolic forms of the propositions; to specify the rules by which proofs may legitimately proceed from step to step, we need only take into account propositions qua sentences, that is, concatenations of symbols. Formality has to do just with what is provable. One proposition follows from another because their forms stand in some specified relation; the content they express is not directly material to this.

What is important to observe here is that for Frege, since a language is a system of signs, it is an interpreted system. This simply follows from the doctrine that sense determines reference. Moreover, it is a uniquely interpreted system since sense 
uniquely determines reference; thus, a change in the system of signs would not just be a different interpretation for the language but a different language altogether. With respect to a particular language, it is just not possible for a sign to have a reference different than what it has. Consider the sign 'Frege'. To Frege, it makes no logical sense to be asked to consider, with regard to some particular language, a circumstance in which its reference is someone other than Frege, for then it would have to express a different sense. This is entailed by the doctrine of sense and reference, for if there are different references, then there must be different senses, and hence we would be considering a different sign. In contrast, insofar as we can talk of symbols (not signs) having reference, there is nothing incoherent in considering circumstances under which they have different reference. The relation of symbol to reference is not determined (it is not mediated by sense) but rather is an arbitrary matter of linguistic convention which could be otherwise. There is nothing inherently nonsensical in considering a circumstance in which the symbol 'Frege' refers to Russell; there is just nothing very interesting or useful in doing so, as far as Frege is concerned, with respect to the project of logic.

We are now in a position to answer the first question we posed regarding $(A x)$. All we need observe is Frege's presumption that propositions too have references, to the True or the False, and because this reference is determined by the thoughts they express, each proposition comes immutably equipped with one and only one truth value. Now we just transpose the argument just outlined-we can make no greater sense of considering a true thought (that is, a thought whose reference is the True) to be false, for to do so would not be to consider that thought, but another. Now, axioms are propositions, so what holds for propositions holds for them. To consider a given axiom to be other than true would be to consider it having a reference other than what it has, and hence as expressing a different thought. This new proposition, however, could not itself be an axiom since its reference would not be the True. The second clause of $(A x)$ we now see is in fact entailed by the first, given Frege's assumption about the nature of language.

For Frege then, a language is an interpreted system as it must be because it is composed of signs. It is, moreover, an inherently interpreted system; to Frege, it would be a non sequitur to speak of an interpreted language in the sense of an interpretation being assigned to a language, for this presupposes something that Frege rejects, that a language itself is a system of meaningless marks or symbols (individuated solely by their shapes). Why does Frege reject this presupposition? At least part of the reason is found in remarks such as the following from his criticism of formalist mathematics in Grundgesetze:

an arithmetic with no thought as its content would also be without possibility of application . ... Why can arithmetical equations be applied? Only because they express thoughts. How could we possibly apply an equation which expresses nothing and is nothing more than a group of figures, to be transformed into another group of figures in accordance with certain rules? Now, it is applicability alone which elevates arithmetic from a game to the rank of a science. So applicability necessarily belongs to it. Is it good, then to exclude from arithmetic what it needs in order to be a science? ([12], §91)

Accepting the presupposition, according to Frege, would prevent the possibility of a substantive arithmetic with a contentful notion of number and hence an arithmetic that is usable and scientific. The point, while perhaps most profound for Frege with 
respect to arithmetic, is obviously more general, applying just as much to geometry; content is needed for applicability. To accept the presupposition about language is thus symptomatic of a deeper confusion about mathematics; it is a confusion that afflicts, in Frege's view, not only the formalist mathematicians he attacks in Grundgesetze (Thomae and Heine being his particular targets) but also Hilbert (although, of course, Hilbert did not think himself confused in the slightest). It is over this issue-whether languages are interpreted or not- that Hilbert and Frege part company.

To see Hilbert's view of language we can look at the source that Frege did, Hilbert's vastly influential Grundlagen der Geometrie [22], first published in 1899. Among Hilbert's achievements in this volume was the first modern example of independence proofs. After giving the axioms of geometry in Chapter 1, Hilbert proceeds in Chapter 2 to show that his axiomatization is optimal, in that none of the axioms is already a consequence of the remaining ones. Hilbert's method is to show that some axiom $A$ is not already a consequence of the set $T$ of the remaining axioms by exhibiting an interpretation of the language (here, of geometry) which makes all axioms in $T$ true but on which $A$ turns out to be false. ${ }^{8}$ (Frege puts it thusly, in his letter to Liebmann of 29 July 1900: "The independence of an axiom $A$ from others is the lack of contradiction between the contradictory of $A$ and the other axioms.") $)^{9}$ To show this, however, Hilbert must crucially assume that a language does not come with a fixed interpretation.

In Chapter 2 of Grundlagen, Hilbert shows the independence of the completeness axiom. This is the axiom that guarantees that the system of points of geometry is maximal, in that it cannot be further extended while still realizing the remaining axioms. To show the independence, Hilbert considers the field of all algebraic numbers that arise from the number 1 by repeated application of the four arithmetic operations together with the fifth operation taking a number $x$ to $\left|\sqrt{\left(1+x^{2}\right)}\right|$. Pairs $(x, y)$ of algebraic numbers are identified with points; the ratios $(u: v: w)$ of any three algebraic numbers are identified with lines; and the incidence relation of a point on a line is given by the condition:

$$
u x+v y+w=0 .
$$

One can then show that under this interpretation all axioms of geometry turn out true, except the completeness axioms, since the field of the algebraic numbers can be extended (consistently with the axioms of geometry) by adding, for example, any nonalgebraic point, and further closing under the five operations.

A similar construction can be used to show the independence of the Archimedean axiom which guarantees, given segments $A$ and $B$, the existence of a number $n$ such that the length of $n$ copies of $A$, laid side by side, exceeds the length of $B$. Consider the field of all algebraic functions (not numbers) of one variable $t$ that arise from $t$ (that is, from the identity function) by the four arithmetic operations together with the abovementioned operation assigning to a function $f(x)$ the function $\left|\sqrt{\left(1+f(x)^{2}\right)}\right|$. Any such function, being algebraic, will have only finitely many zeros, and therefore it will be eventually always positive or always negative for sufficiently large arguments. We can then compare two such functions $a$ and $b$, and put $a<b$ whenever $b-a$ is eventually positive. On this understanding, if $n$ is any positive integer, then we have $n<t$ (since the difference $n-t$, considered as a function of $t$, is eventually always negative). But this shows that there are numbers, namely, 1 and $t$, such that no multiple of the first can exceed the second. Since it is also possible to show, as 
before, that the other axioms are satisfied, it follows that the Archimedean axiom is independent of the other axioms.

These examples are sufficient to show that Hilbert's conception of independence proofs could not have been more alien from Frege's point of view. As we have seen, Hilbert's independence proofs rest on the possibility of reinterpreting the axioms of geometry by appropriately changing the meaning of such expressions as 'point', 'line', 'incidence', and so on. In the proof of independence of the completeness axiom, Hilbert reinterprets 'point' as meaning 'pair of algebraic numbers' and 'line' as meaning 'ratio of the three numbers $u, v, w$ '. Here we observe an assumption that will become a hallmark of the modern model-theoretic approach to language and interpretation. On this assumption, a language is a system of uninterpreted symbols, so that a language is to be regarded as a set of sentences (in Frege's sense, that is, in contrast to his use of 'proposition'). A language understood in this manner can be interpreted in a variety of ways, allowing one to consider general questions such as what would obtain under all interpretations, or all interpretations of a certain sort. Hilbert's allowing for reinterpretations makes sense just under this sort of understanding of language. If language is inherently uninterpreted we may consider it now under some given interpretation and then under some other.

But this is totally inconceivable from the point of view of Frege's conception of language as interpreted. As we have seen, language for Frege is a system of signs where a sign is a pair made up of a symbol and a sense. If we were to assign a different sense to one of the signs in the language, then we would have a different language. There is no room in Frege's conception for the notion of an alternative interpretation for a given language; indeed "The word 'interpretation'," Frege says, "is objectionable, for when properly expressed, a thought leaves no room for different interpretations." Frege elaborates as follows:

As if it were permissible to have different propositions with the same wording! This contradicts the rule of unambiguousness, the most important rule that logic must impose on written or spoken language. If propositions having the same wording differ, they can do so only in their thought-content. Just how could there be a single proof of different thoughts? This looks as though what is proved is the wording alone, without the thought-content; and as though afterwards different thoughts were then supposed to be correlated with this wording in the different disciplines. Rubbish! A mere wording without a thought-content can never be proved. ([14], pp. 79-80)

A "purely formal system" a la Hilbert thus suffers a fatal flaw—we cannot conduct proofs within it. In a proof, "Each of the premises is a determinate thought recognized as true: and in the conclusion, too, a determinate thought is recognized as true. There is here no room for different interpretations" ([14], II, p. 82). Hilbert's system is thus not even logic, and so it is no surprise that Frege thought that no sense could be made of the status of propositions in such systems since they do not contain any propositions in the first place. That Hilbert confuses such fundamental notions as axiom and definition is in turn not unexpected since, strictly speaking, there are no extensions of these notions in a purely formal system and so there are no principled grounds in such a system for drawing the distinction. According to Frege, when Hilbert makes remarks such as "The axioms of this group define the concept of 'between' . . ", he compounds in a mere ten words two mistakes. First off, concepts are not the sort of things that are defined, but even more egregiously, Hilbert, in speaking of axioms 
as defining, has conflated two quite distinct things. Axioms, by Frege's light, are true propositions of a certain sort (assertable, if not provable) and are to be sharply distinguished from definitions. Definitions give, by stipulation, meaning to terms that do not have any; they provide an interpretation for an uninterpreted symbol, introducing the definiens into the language as a meaningful sign that can then be used as a propositional constituent. (Definitions for Frege are thus explicit and abbreviatory.) Per se, definitions are neither true nor false for they are not fully concatenations of signs and hence cannot be asserted; they are not propositions (although once the definiens has been introduced, an identity statement expressing the definition can then be asserted since it will contain all and only signs). This cleavage between propositions and definitions for Frege is sharp and absolute; one cannot do the job of the other. In particular, axioms, by their very nature, cannot define, for what is there in an axiom, a proposition, to define? Frege thus rejects implicit definition and to hold otherwise, so that axioms can define, is thus something of a category mistake; certainly the burden would be on someone who wishes to speak in this novel way about axioms to show why it is not a hopeless confusion. Frege says: "I believe that with my exposition about the use of the words 'axiom' and 'definition' I move within the bounds of traditional usage, and that I may justifiably demand that one not cause confusion by a completely new usage of these words". 10 By this usage, Frege says that "What I call a proposition tout court or a real proposition is a group of signs that expresses a thought; however, whatever only has the grammatical form of a proposition I call a pseudo-proposition" ([14], p. 69). Hilbert, as Frege sees it, traffics in pseudo-propositions, not real propositions.

Insofar as Hilbert's sentences have a modicum of propositional status, it is thus, according to Frege, as pseudo-propositions. But so understood, what is to be made of them? Not much, according to Frege, and certainly not much in the context of proving the independence of the parallel axiom. Hilbert outlines the proof in the following passage:

The axiom of parallels IV is independent of the other axioms. This is most simply shown in a well-known way as follows: Let the points, lines, and planes of the ordinary (Cartesian) geometry constructed in Section 9, which lie in a fixed sphere be chosen as the elements of a space geometry and let the congruences of this geometry be replaced by linear transformations of ordinary geometry that map the fixed sphere into itself. By suitable interpretations it can be seen that in this "non-Euclidean" geometry all axioms except Euclid's axiom IV are valid and since the existence of ordinary geometry has been proved in Section 9 the existence of non-Euclidean geometry follows now. ([22], pp. 32-33, Hilbert's emphasis)

In and of itself the passage from Hilbert makes no sense to Frege, for it is not making a coherent claim about anything that can claim the status of an axiom:

Mr. Hilbert raises the question whether axioms are independent of one another, and then continues "indeed, it turns out that none of the axioms can be deduced from the remaining ones by means of logical inference." According to this, he appears to use the word "independent" just as has been stipulated above. But apparently it only seems that way, since in our case we are concerned with thoughts; Mr. Hilbert's axioms, however, are pseudo-propositions which therefore do not express thoughts. This may be seen from the fact that according to Mr. Hilbert an axiom now holds, and now does not. A real proposition, however, expresses a thought, and the latter is either true or false; 
tertium non datur. A false axiom-where the word "axiom" is understood in the proper sense-is worthy of exhibition in Kastan's Waxworks, alongside a square circle. ([14], p. 104)

Showing something about pseudo-propositions is not to prove anything at all about real propositions, certainly not the independence of axioms. A "proof" that holds with respect to pseudo-propositions is no proof at all, and this to Frege undermines Hilbert's approach to independence proofs. Frege makes this abundantly clear in the following passage excoriating Hilbert:

It must be noted that Mr. Hilbert's independence-proofs simply are not about real axioms, the axioms in the Euclidian sense; for these are surely thoughts. Now, nowhere in Mr. Hilbert's writings do we find a differentiation that might correspond to our own between real and pseudo-propositions, between real and pseudo-axioms. Instead, Mr. Hilbert appears to transfer the independence putatively proved of his pseudo-axioms to the axioms proper, and that without more ado, because he simply fails to notice the difference between them. This would seem to constitute a considerable fallacy. ([14], p. 102)

Frege continues this remark by elucidating what he sees as the cause of the fallacy, in an ambiguity in the way that Hilbert has employed the fundamental geometrical notions:

The fault here lies in the double usage of the words 'point', 'straight line', and so on, which on the one hand, like letters are to lend generality to the whole theory, in which case they do not designate anything; and on the other hand have their traditional references in the Euclidian axioms. In the former case his axioms are merely pseudo-axioms without sense, since only the whole. . . whose dependent parts they are, has a sense-in which case the Euclidian axiom of parallels simply does not occur, and consequently nothing can be proved of it. In the other case real axioms do occur. But then these independence-proofs are inappropriate, since it is impossible to substitute other concept-words for "point", "straight line", and so on. But surely it is on the very possibility that such a proof depends. ([14], p. 102)

If Hilbert's propositions are to be understood as real axioms, then all the terms they contain must have predetermined meanings. This would be precluded if the terms 'line', 'point', 'plane', and so on were to have variable meanings; it makes no sense, on Frege's view to speak of "reinterpretations" of these terms: "unless we are to understand the words in the Euclidean sense, just about everything is unknown" (p. 90). But this is to rule out the key move Hilbert needs to prove independence.

The alternative that Frege mentions is to understand Hilbert as using the geometric terms as (free) variables, "letters" in Frege's terminology. ${ }^{11}$ But while we will then only have pseudo-propositions which express no thoughts (as they are not about determinate objects) we now have a wedge, Frege thought, to rendering the mathematical content of Hilbert's observations, and he devotes the second section of [14] to recasting Hilbert in this light. The strategy is to take Hilbert's pseudo-axioms as antecedents of conditionals whose consequents are pseudo-theorems and then taking their universal generalizations, the bound positions corresponding to the positions in which Hilbert uses geometric terms. The resulting proposition does express a thought; it is a real proposition, although none of its component parts are. With respect to such universal generalizations, Hilbert's reinterpretations now become specimens of inference from general to particular; they are to be understood, according to Frege, as varying instantiations of the variables with meaningful terms, resulting in conditionals whose 
clauses are real propositions. Now suppose that we instantiate the variables with the Euclidian notions; the antecedent of the conditional so derived will then consist of one, or the conjunction of some, Euclidian axioms. Since the Euclidian axioms are true, the consequent may be detached, although this may not be possible on other instantiations. If it is not, we are left with nothing more than a hypothetical proof.

Against this understanding of Hilbert, Frege elucidates what he thinks Hilbert has demonstrated by his independence proofs. Let $A_{1} \wedge \cdots \wedge A_{n-1}$ be the conjunction of Hilbert's "axioms" save the parallel axiom, and let $P$ be the parallel axiom. Then what Hilbert shows is that the higher-order generalization:

$$
\forall F_{1}, \ldots, F_{n}\left(A_{1} \wedge \cdots \wedge A_{n-1} \supset P\right),
$$

where at least one of $F_{1}, \ldots, F_{n}$ shows in each of $A_{1}, \ldots, A_{n-1}, P$, is false by exhibiting an instance that has a true antecedent but a false consequent. Frege remarks on the significance of this as follows:

Hilbert's independence-proofs concern neither the independence of propositions in the sense just recommended, nor the independence of thoughts. Rather, they concern the independence of the references of the parts of pseudopropositions. These parts are the largest that still refer. But they are not propositions, and therefore do not express thoughts. ([14], p. 101)

In this remark, Frege indicates both what is and what isn't shown on his understanding of Hilbert's demonstration. What is shown is the independence of certain secondlevel concepts; namely, $A_{1}(\xi) \wedge \cdots \wedge A_{n-1}(\xi)$ and $P(\xi)$, the concepts that can be extracted from Hilbert's pseudo-axioms. These are the references of the largest parts of the antecedent and consequent clauses that have determinate meaning. What is not shown is the independence of the parallel axiom itself, where "axiom" is meant in Frege's sense of a "real" proposition, one that expresses a thought. "Mr. Hilbert's independence-proofs simply are not about real axioms, the axioms in the Euclidian sense; for these, surely, are thoughts" ([14], II, p. 102). As we shall see, according to Frege something different is needed to establish results about these. ${ }^{12}$

\section{Externalism versus Internalism}

While it is to speak the obvious to say that with Frege modern logic begins, nevertheless at certain very significant points Frege's conception of logic sharply varies from the modern conception in ways that do not easily translate over to the contemporary viewpoint. One place, perhaps the central place, where this is so is in Frege's insistence on language as an interpreted system, along with all that assumption entails (cf. Frege's adherence to proposition $(A x)$ ). Where Frege in his critique parts company with Hilbert is where Frege parts company with modern logic, in which the assumption that language is an uninterpreted system has been at the heart of metatheoretical and model-theoretic results about logic. But in diverging from modern logic in this way, does Frege also diverge from these results? That is, was Frege precluded from being metatheoretical about logic because of his assumptions about language? To answer this we turn to the issue of metatheory in Frege's logic.

First, let us distinguish between two perspectives toward logic, internalism and externalism. By internalism we mean the view that logic is exhausted by the practice of deriving consequences. Externalism, on the other hand, allows for the possibility of taking a logical system as an object of formal investigation itself. (In Belnap's apt phrasing, this is the distinction between the art and the science of logic.) The 
internalist perspective toward logic is, of course, undeniable in contrast to the externalist perspective; one can use logic to prove theorems without any conception that the ways by which logic does this can itself be the locus of inquiry. It is widely thought that Frege, and Russell as well, were impelled by their very conceptions of logic to take solely the internalist perspective and that this closed for them the door to anything akin to contemporary metatheory. There is without doubt more than a nugget of truth to this; Frege did not have metatheory as we know it. The reason for this, however, is not a rejection of externalism per se, but rather rejection of a central tenet of a version of externalism. To be sure, this rejection severely limits the extent of metatheoretical reasoning available to Frege but it does not exclude for Frege an externalist perspective on logic altogether.

The externalist perspective as we are conceiving it is neutral in both a syntactic and a semantic sense. In the syntactic sense, externalism is neutral with respect to the question whether the metalanguage, that is, the language in which propositions about the logic qua object language are formed, is distinct from the object language itself. That is, externalism is neutral as to whether a language can be used to talk about itself. In the semantic sense, externalism is neutral on the necessity of being able to reinterpret terms of the language. So, as we understand externalism it is not to prejudge two characteristics that we naturally associate with the contemporary view of metatheory stemming from the classic ideas of Tarski: the first is the idea that object language and metalanguage are distinct; and the second is the idea that metatheory is model-theory, that is, the theory of alternative interpretations. Metatheory, however, insofar as it is to be equated with externalism, is a more general notion than Tarski's. On our view, the necessary step for obtaining metatheoretical results—soundness, completeness, independence, and so on-is externalism. The open question is whether we also need to make the narrower assumptions embedded in a Tarskian metatheory. Do we need metatheory so construed in order to metatheoretically reason so as to obtain metatheoretical results? Put differently, while metatheory in this sense is sufficient to obtain metatheoretical results, as Tarski famously taught us, is it also necessary? Frege's answer is that it is not.

To understand Frege's answer, we need to consider in a bit more detail Frege's view of logic. Frege begins by assuming that there is a single fixed universe, composed of functions and objects, and that there is a language (Begriffsschrift) in which propositions about the universe can be formed. Moreover, Frege assumes that the language is fully expressive relative to the universe, in the sense that a sign can be formed for every sense that can be grasped. With respect to this system, certain propositions may be designated as axioms, the result being, when supplemented with rules of inference, what we referred to in note 6 as a logistic system. Logic per se will be the system that contains just the logical axioms, that is, the six Basic Laws of [12]. ${ }^{13}$ Truths that can be proven using the specified rules of inference from these axioms (perhaps with the help of explicit definitions) are logical truths. ${ }^{14}$ Frege's logicism is the thesis that if we take the resources needed to characterize reasoning in general, that is, logic as we have just described it, then we are in possession of sufficient resources to characterize arithmetical reasoning, that is, to prove all the truths of arithmetic. ${ }^{1 .}$ Arithmetic truths, therefore, are logical truths. Now, arithmetic truths are truths about the subject matter of arithmetic, namely, numbers; hence, truths about numbers are logical truths. But numbers for Frege are logical objects, by which he means that they 
are definable given the aforementioned resources and nothing more. Consequently, such truths about logical objects are, for Frege, logical truths.

Within the language, that is, within the conceptual notation (Begriffsschrift), it will be possible to form all sorts of propositions that are not logically provable in that they do not follow from the logical axioms. They may, however, be provable in some application of logic. For Frege, logic is applied by the addition of new axioms which extend logic to further domains of content by making possible proofs of more propositions about the universe which is itself a constant across all applications. These new axioms will be justified in ways pertinent to their domain of applicability, the most notable example discussed by Frege being the axioms of geometry which are justified by geometric intuition. Thus, the truths provable in any given application will bifurcate into logical and nonlogical truths; which a given truth is will depend upon whether its proof follows solely from (substitution instances) of the axioms of the core logic or depends upon at least one of the special axioms of the application. So, for example, 'Opposing angles are equal' is a nonlogical truth of geometry, but 'Opposing angles are equal or opposing angles are not equal' is a logical truth.

Notice that Frege's conception of logic incorporates a certain notion of universality in the sense that within the Begriffsschrift propositions can be formed about everything in the universe. Now the question arises as to whether this includes propositions about logic itself? At one level, the answer seems straightforward; why wouldn't logic be part of everything there is? As Tappenden [34] neatly puts it, "if logic is universal - if its scope includes absolutely everything-one would normally expect that this should include logic itself." Frege, we know, allowed in the Begriffsschrift expressions that refer to expressions (cf. the discussion of identity of content in Frege [10], §8) and hence for propositions expressing thoughts about the language. But this, while necessary for logic to be about itself, is not sufficient if such propositions are to be metatheoretically significant. To achieve this status, the propositions must either be axioms or theorems; but to show this, we must have an application of logic whose content area is logic itself. Such an application, in which propositions about logic would be proven from axioms about logic, would be possible on Frege's view of logic, given the generality of its applicability. The application would constitute a Fregean metatheory.

Given this, it is with some degree of curiosity that we discover remarks in the literature that invoke the universality of Frege's logic as implying exactly the opposite conclusion, namely, that any such application is impossible. What are we to make of comments such as the following, due to Dreben and van Heijenoort?

For Frege, and for Russell and Whitehead, logic was universal: within each explicit formulation of logic, all deductive reasoning, including all of classical analysis and much of Cantorian set theory, was to be formalized. Hence, not only was pure quantification theory never at the center of their attention, but metasystematic questions as such could not be meaningfully raised. We can give different formulations of logic, formulations that differ with respect to what logical constants are taken as primitive, or what formulas are taken as formal axioms, but we have no vantage point from which we can survey a given formalism as a whole, let alone look at logic whole. . . The only way to approach the problem of what a formal system can do is to derive theorems. ([6], p. 44)

In order for metatheoretical questions to be raised, "the Frege-Russell-Whitehead view of logic as all embracing had to be abandoned, and Frege's notion of a formal 
system had to become itself an object of mathematical inquiry and be subjected to the model-theoretic analyses of the algebraists of logic." ${ }^{18}$ This line of thinking is echoed in remarks by Goldfarb: "if the system constitutes the universal logical language, then there can be no external standpoint from which one may view and discuss the system. Metasystematic considerations are illegitimate rather than simply undesirable" [17]. The thesis here is a very strong one; because Fregean logic is universal, because its universality leaves no room for "standing outside" of logic, of adopting an "external" perspective, no metatheoretical questions can in principle even be broached. There can be no Fregean metatheory.

The difficulty with this thesis, that universality implies no metatheoretical reasoning, is that it is extremely unclear how to make it square with Frege's conception of logic, for if one is going to deny that Frege has any access to metatheory, even in our very general sense of externalism, then in fact what one precisely has to do is deny the universality of Frege's logic. In order to say that Fregean metatheory is impossible one of two things must be maintained. Either propositions about logic are unformulable in the Begriffsschrift or, if they are formulable, then given the full class of applications, in no application could such propositions be either axioms or theorems. But to hold the former is to deny that propositions can be formed about everything in the universe, while to hold the latter is to deny the generality of the applicability of logic. Thus, it must be some other thesis that is meant regarding how universality restricts the scope of Fregean logic. What might that be?

Recall that what is true of Frege's conception of logic is that a Tarskian-style metatheory is outside its purview, and there is at least general consensus about the proximate cause; as we have discussed, from Frege's view of language as an interpreted system it follows that propositions do not allow for reinterpretation, and this is sufficient to eliminate such a conception of metatheory. Goldfarb puts the point as follows: ${ }^{19}$

For Frege and Russell the propositions of logic contain no nonlogical vocabulary: there are no schematic placeholders which may be assigned one value or another. Every logical formula has a fixed meaning; there is no question of reinterpreting any sign.

Perhaps then there is a weaker thesis that is intended regarding the universality of Frege's logic, that it implies that there can be no reinterpretation of the nonlogical constants. So consider the two notions of universality we have at play-that logic is about everything and that its applicability is completely general. A natural corollary of these properties of logic is that the truths of logic are the most general sorts of truth, ones that hold of everything and across all applications. Goldfarb elucidates his understanding of Frege's take on this: "for Frege . . logic is about something, namely, everything. The laws of logic have content: they are the most general truths about the logical furniture of the universe." This is to be contrasted with the modern view, which Goldfarb characterizes as follows:

Our view of logic carries with it the notion that logical truths are completely general, not in the sense of being the most general truths about logical furniture, but rather in the sense of having no subject matter in particular, of talking of no entities or sorts of entities in particular, and of being applicable no matter what things we wish to investigate. ([17], pp. 352-53)

For Frege, since logical truths are truths about something - the logical furniturewe cannot reinterpret them to be about anything else, as opposed to modern logic 
which operates under no comparable stricture. Moreover, although Goldfarb does not mention this, it also appears to follow that any truths about logic derived in a Fregean metatheory (which is not precluded on the present view) would not be logical truths, for they are not about the logical furniture. ${ }^{20}$

The issue appears transformed. It is now about Frege's view of logical truth; universality has been put to the wayside, for if logical truths, in being truths about the logical furniture, are to be universal truths, it must be because whatever holds of logical objects, holds of all objects. (Short of such a relation, logical truths would not be truths about everything, but only about some things.) But clearly it is not Frege's view that every logical truth is a truth about logical objects. It follows from the logic of [12] that 'Frege wrote Grundlagen der Geometrie or Frege didn't write Grundlagen der Geometrie' is no less a logical truth than ' $2+2=4$ or $2+2 \neq 4$ ', yet only the latter contains thoughts that are about logical objects. What is true of Frege's view of logical truth is the converse: every truth about logical objects is a logical truth. But, it is hard to see what follows of interest from this, for one could agree to the truth of this statement, and still hold the "modern" view of logical truth (true in all models) by merely holding the view that there are no purely logical objects. Even Frege would feel little encumbrance with respect to his view of metatheory, since it would leave open whether truths about logic are logical truths; as noted, this would be a matter of the nature of the axioms of that application.

Let's take stock. There is a property that all logical truths have, but it is not a property that distinguishes them from nonlogical truths. It is that they are all propositions in Frege's sense, and hence inherently contentful, that is, express thoughts. As we argued in the previous section, this is sufficient, given Frege's conception of language as an interpreted system with its roots in the doctrine of sense and reference to rule out a conception of metatheory that requires us to be able to consider alternative interpretations for a given language. If we are seeking a locus of the difference between Frege's view of logic and the modern views, we find it here in this incompatibility with a model-theoretic conception which formally requires an uninterpreted language. Frege would not have seen modern metatheory as a valid application for just the reasons that he criticized Hilbert; it would be an application without subject matter, for one cannot study, in Frege's view, the form of a thought without also studying the thought itself; their relation is too intimate. In this regard, one could not "step out" of the system and adopt a semantic stance toward it. Frege's view, however, is not incompatible with metatheory tout court, externalism in our sense. Because Frege's logic is universal, there can be an application that has propositions as its subject matter; establishing metatheoretical results within this application would be to prove, within the language itself, theorems about the propositions. ${ }^{21}$ But what sorts of metatheoretical results can we expect to obtain in such a metatheory, of which we have spoken thus far only in the most general terms? It is to this question we now turn, in explicating Frege's New Science.

\section{Frege's New Science}

Frege's New Science can be contrasted with his "old" science just as the difference we have drawn between the externalist and internalist perspectives toward logic. The old science is concerned with establishing propositions either as axioms or via proofs within the Begriffsschrift; the New Science is concerned with relations between propositions so established. The New Science arises from the old by the addition of 
new axioms, and is applied by Frege to the question of "whether, taking Hilbert's results as a starting point, we might not arrive at a proof of the independence of the real axiom" (see [14], p. 103). The third part of the second series of Frege's [14] is devoted to showing how, within the New Science, this result can be obtained.

Frege frames the issue as follows:

Is it possible to prove the independence of a real axiom from a group of real axioms? This leads to the further question: how can one prove the independence of a thought from a group of thoughts? First of all, it may be noted that with this question we enter into a realm that is otherwise foreign to mathematics. For although like all other disciplines, mathematics, too, is carried out in thoughts, still, thoughts are otherwise not the object of its investigation. Even the independence of a thought from a group of thoughts is quite distinct from the relations otherwise investigated in mathematics. We may assume that this new realm has its own specific, basic truths, which are as essential to the proofs constructed in it as the axioms of geometry are to the proofs of geometry; and that we also need these basic truths especially to prove the independence of a thought from a group of thoughts. ([14], p. 106)

This passage raises the following issue: what does Frege mean by the independence of thoughts (and hence of propositions) and then, given this notion, what new laws are needed in order to prove the independence of a thought from other thoughts? Frege's answer directly ties the notion of independence to derivability. A thought depends on some set of true thoughts if and only if it is provable from that set through a sequence of logical steps. If it is not so derivable it is independent of them. (Note the difference from the modern notion, according to which a proposition $A$ is independent of a group of thoughts $G$ if it is neither provable nor refutable on the basis of $G$; for Frege if $A$ were refutable from $G$ it would still be independent of $G$.) Given this notion of independence, Frege then gives the new law needed to prove independence, presenting it in the form of a procedure which if successfully carried out conclusively establishes the independence of a thought.

More precisely, Frege asks us to consider, in Section 3 of [14], a double list of words, in which each expression is paired with one of the same logical type: proper names to proper names, concept-words of a given level to concept-words of the same level, and so on. In keeping with Frege's conception of interpreted languages, each word, whether listed on the left or on the right has definite sense and reference. The list is assumed to be one to one, in that neither on the left nor on the right is the same word listed twice, ${ }^{22}$ and, moreover, it is assumed that words "whose references belong to logic" are paired to themselves.

If a listing satisfying the above-mentioned requirements is given, then one can consider the associated translation of each proposition $\varphi$ into a proposition $\varphi^{\prime}$ obtained by replacing each word in $\varphi$ by the word paired to it in the listing. Then it is possible to establish the independence of a thought $G$ from a group $\Omega$ of thoughts by exhibiting a permutation of the nonlogical vocabulary that keeps the truth values of thoughts in $\Omega$ fixed while changing the truth value of $G$. In other words, if $\pi$ is a permutation of the nonlogical vocabulary, and $G^{\prime}$ and $\Omega^{\prime}$ are the results of applying $\pi$ to $G$ and $\Omega$, respectively, then $G$ is independent of $\Omega$ if and only if there is a permutation $\pi$ under which $G^{\prime}$ is false while each member of $\Omega^{\prime}$ is true. Now, suppose that $\Omega$ is the set of Euclidian axioms less the axiom of parallels, and $G$ is the axiom of parallels, and that $\Omega$ maps onto $\Omega^{\prime}$ by a permutation that replaces expressions for points and 
lines of Euclidean plane geometry by expressions for the points and lines that lie in a fixed sphere, and maps ' $x$ is congruent to $y$ ' of plane geometry onto ' $x$ is taken into $y$ by a linear transformation of the sphere into itself'. As is well known, under this permutation the axiom of parallels fails, while all the other axioms of Euclidean geometry are still true; consequently, by Frege's method this "Hilbert" permutation establishes the independence of the parallel axiom.

It is important to observe here that Frege's method for establishing the nonprovability of the parallel axiom does not presuppose any reinterpretation of primitive geometric terms; in establishing independence, no proposition is assumed to have any meaning other than what it in fact has. No true proposition is assumed to be false. $^{23}$ This is in keeping with Frege's conception of logic as an interpreted system. Moreover, in establishing this fundamentally metatheoretical result, Frege has not "stepped outside" of the object language — the method or permutation holds within the object language itself. It thus conforms to the externalist perspective toward logic, and in this regard Frege's New Science is metatheoretical.

Frege is not without some degree of skepticism toward the New Science, however. In part this reflects a natural degree of caution due to the sketchy and informal nature of his remarks:

With this, we have an indication of the way in which it may be possible to prove the independence of a real axiom from other [real] axioms. Of course, we are far from having a more precise execution of this. In particular, we will find that this final basic law, which I have attempted to elucidate by means of the above-mentioned vocabulary still needs more precise formulation and that to give this will not be easy. ([14], p. 110)

But there is a more fundamental sort of worry for Frege: "Furthermore, it will have to be determined what counts as a logical inference and what is proper to logic." The worry here is with the logical terms. As we have seen the permutation argument presupposes that in the given double list so-called logical words be paired with themselves so that logical form is preserved; this is in order that proofs be mapped into proofs by the given permutation:

In order to be sure that in our translation, to a correct inference on the left there again correspond a correct inference on the right, we must make certain that in the vocabulary to words and expressions that might occur on the left, and whose references belong to logic, identical ones are opposed on the right. ([14], p. 110)

But as Frege's logic stands, the characterization of the logical words is by stipulation: there is no general method by which we can split the logical and nonlogical vocabulary. Of course, since the inventory of Frege's logical terms corresponds to the classical truth functional connectives and quantifiers, his argument for independence, with these held constant, has considerable intuitive appeal. Nevertheless, in order for Frege's method to be reliable, we must have some way of knowing that these logical terms are all the logical terms. For otherwise there might be logical terms beside the ones stipulated that are mistakenly permuted, in which case some thoughts might incorrectly turn out to be independent of other thoughts. Without a characterization of the logical terms, if the permutation is conceived as an independence test, it would thus be possible to have false positives (but not false negatives, given that the terms that Frege identifies as logical are, in fact, logical). It is thus clear that for Frege's approach 
to be successful, it needs to rest on the solid foundation of a general, independent characterization of logical constants.

Thus the question we arrive at is the following: in the context of Frege's New Science can we go beyond merely stipulating what the logical terms are and give a general characterization of the logical part of the vocabulary?

\section{Toward a Fregean Characterization of Logical Notions}

In a posthumously published paper ${ }^{24}$ Tarski elaborated a generalization of Klein's famous Erlangen Program for the classification of geometric notions. Just as Klein proposes to identify geometric notions as those that are invariant under a certain class of transformations, Tarski also wants to characterize the logical notions as those that are invariant under all permutations of the domain. Tarski's proposal, which has been elaborated especially by Sher and $\mathrm{McGee},{ }^{25}$ requires that formulas be identified with the sets of assignments to the variables of the language that satisfy them, and unary connectives with maps from sets of variable assignments into sets of variable assignments, and similarly for $n$-ary connectives. Once this identification is carried out, any permutation of the domain of quantification can be "lifted," first to variable assignments, then to sets of variable assignments, and finally to maps taking sets of variable assignments into sets of variable assignments. The logical notions of each type can then be characterized precisely as those that are left unchanged by every permutation. The familiar operations of conjunction, disjunction, negation, existential or universal quantification, and so on all turn out to have a logical character on this proposal because they are unchanged by every permutation of the domain of quantification. Similarly, all cardinality quantifiers of the form, for example, 'there exist uncountably many' are also logical, as permutations preserve cardinality.

Although a characterization of the logical terms under the specific sorts of assumptions that underlie the Tarski-Sher-McGee approach is unavailable to Frege (given the former's dependence on a conception of metatheory that incorporates modeltheoretic notions) one is nevertheless struck by the similar method of permutation in the Tarskian characterization of the logical terms and Frege's method in [14] for showing independence. That is, while they differ in that on Frege's approach what are permuted are expressions of the language, not individuals of the domain, both can be seen as building on Klein's Erlangen Program. ${ }^{26}$ As the Erlangen program aims to characterize and classify geometric notions through their invariance under certain transformations, so do both the Tarskian and Fregean approaches aim to characterize logical notions through invariance conditions. ${ }^{27}$ We are thus tempted to see whether Frege's method can be generalized to provide a parallel account to Tarski's of the characterization of the notions that are to be counted as logical, so as to generate, in terms congenial to Frege, a general conception of the logical constants.

Our approach to a Fregean conception of logical constants consists of three distinct steps. In the first step we assume that we have a permutation $\pi$ of the vocabulary of the language. As before, the permutation is assumed to preserve grammatical categories: object-names are mapped to object-names, concept-words to concept-words, and so on. Since Frege's Begriffsschrift framework is higher-order, we also require that firstlevel concept-words be mapped into first-level concept-words, second-level conceptwords into second-level concept-words, and so on. ${ }^{28}$ We also assume that atomic names for truth values are mapped to atomic names for truth values. (Thus, if "True" and "False" are the only atomic names for the truth values, there can be only two 
kinds of permutations: those that map the truth values to themselves and those that switch them.) Each such permutation $\pi$ gives rise to a corresponding permutation $\pi^{*}$ on propositions where $\pi^{*}$ takes, for example, a proposition $C a$ into the proposition $\pi(C) \pi(a)$. Where $\varphi$ is a proposition, we will just write $\pi(\varphi)$ for $\pi^{*}(\varphi)$. Notice that, so far, we are still completely within the framework Frege describes for independence proofs in geometry. The second step in the argument consists in the identification of a class of permutations, where any such class gives rise to a corresponding notion of logical truth. In fact, once we have such a class of permutations, we can then simply identify the logical truths as the following set, where $d(\alpha)$ is the reference of $\alpha$ :

$$
\{\varphi \mid \forall \pi, d(\pi(\varphi))=d(\pi(\text { True }))\} .
$$

That is, the logical truths are those propositions $\varphi$ such that $\varphi$ is mapped to the true if and only if the true is mapped to the true, and similarly for the false, given that truth values are mapped to truth values. (We always assume at least the identity permutation, so that in particular, all logical truths are true.) The third step in the argument consists in the identification of logical constants. Any class $C$ of basic terms (including connectives, quantifiers, and variables of any order) will be a candidate class of logical constants if they are a largest class of terms such that every proposition in that vocabulary, if true, is logically true. ${ }^{29}$

The crucial part of the strategy is in the identification of the appropriate class of permutations. In particular, in identifying such a class, we will look at the way that permutations of names (object-words) and predicates (concept-words) are related to each other. The first and most obvious option is not to impose any restrictions and allow any permutation at all that respects grammatical categories. This liberal policy in admitting permutations results in an overly restrictive notion of logical truth. Recall that for Frege all words are either names or predicates, and that in particular what we now regard as logical constants are just particular kinds of predicates: negation is a predicate of truth values, implication is 2-place relation of truth values, quantifiers are higher-level predicates, and so on. But if this is so, then the liberal permutation policy would allow, say, for the permutation of 'and' and 'or' turning propositions of the form $\ulcorner P$ or not- $P\urcorner$ into falsehoods. This strategy would therefore not even provide for the classical logical constants. We could, of course, require that permutations fix logical constants. But while this might have been an option available to Frege in the context of independence proofs, it is not, on pain of circularity, available to us in the present context. ${ }^{30}$

An alternative class of permutations can be identified by requiring that all permutations in the class satisfy the following condition for any concept $C$ and name $a$ :

(Comm) $\quad C$ applied to $a$ refers to $d$ (True) iff

$$
\pi(C) \text { applied to } \pi(a) \text { refers to } d(\pi \text { (True)). }
$$

By this condition, if $C$ applied to $a$ refers to the True (i.e, if $d(a)$ falls under $d(C)$ in the Fregean sense) then $\pi(C)$ applied to $\pi(a)$ refers to $\pi$ (True), and conversely. Assuming this, since the map $\pi$ is clearly defined for each name in the language, $\pi$ can be limited to the object-names, for this will induce a corresponding mapping on predicates, in which each predicate $\Phi$ is mapped to a predicate $\Phi^{\prime}$ such that $d(a)$ falls under $d\left(\Phi^{\prime}\right)$ if and only if $a=\pi(b)$, for some $b$, and $d(b)$ falls under $d(\Phi)$. (Comm) thus amounts to the requirement that for each predicate symbol $\Phi, \pi(\Phi)=\Phi^{\prime}$, and similarly for predicates of every level of any number of arguments. Here we need to 
assume that the language is expressive enough: in particular, we need to ensure that for any predicate $\Phi$ the clause defining the corresponding predicate $\Phi^{\prime}$ is expressible in the language, and that, moreover, we have all the needed instances of a closure principle of the form (for every formula $\Theta\left(X_{1}, \ldots, X_{n}\right)$ with $X_{1}, \ldots, X_{n}$ variables of the appropriate level):

$$
\exists P \forall X_{1}, \ldots, X_{n}\left(P\left(X_{1}, \ldots, X_{n}\right) \Leftrightarrow \Theta\left(X_{1}, \ldots, X_{n}\right)\right) .
$$

But notice that this schema is a theorem of higher-order logic, and hence a schema that Frege would not have objected to.

The restriction to permutations that commute with predication in this way is rather drastic. Here, it will help to conceive of such permutations in set theoretic terms, as the analogue of automorphisms of the given set-theoretic universe. It follows, of course, that such permutations always map truths to truths and that therefore all truths turn out to be, according to our proposal, logical truths. There is perhaps a way to justify such an account of logical truths in Fregean terms. Let us refer to any universe of entities in which

(a) every object is a course-of-values-including the two truth values; and

(b) Basic Law V holds when restricted to concepts having courses-of-values, as a pure Fregean universe.

It is worth pointing out that this is not an inconsistent notion: pure Fregean universes can be identified with set-theoretic universes satisfying the axiom of extensionality (they only become inconsistent, of course, when we also assume certain unrestricted closure conditions such as that every concept has a course-of-values). ${ }^{31}$ Permutations that unrestrictedly commute with predication can be regarded as automorphisms of the pure Fregean universe. Over such a universe, all true propositions are logically true and therefore all terms are logical terms. (In fact, it is not implausible to think of the objects of such a universe as logical objects.) This is not a scenario that Frege would have found unappealing. Certainly numbers are logical objects for Frege and truths concerning them are logical truths; something analogous might have been the case for courses-of-values or, as we have been thinking of them, sets.

Although we do not live in a pure Fregean universe, since not all objects are logical objects, these considerations do suggest an intermediate condition on permutations that is liberal enough not to vouchsafe all truths as logical truths, but not so liberal that, for example, $\ulcorner P$ or not- $P\urcorner$ turns out not to be a logical truth. The idea here is as follows. Assuming that the two truth values are themselves courses-of-values (as Frege would have liked), (Comm) holds only for those propositions in which all object-names refer to courses-of-values. The effect of this condition is to consider a pure Fregean universe as embedded within a larger domain of objects, and restrict permutations to obeying (Comm), but only with respect to those propositions that are "about" logical objects. ${ }^{3}$

On the basis of this proposal, as long as $a$ denotes some green object, not a courseof-values, we are allowed to map it onto $b$, denoting a blue object while keeping the predicate 'is green' fixed so that the truth ' $a$ is green' is mapped to a falsehood and fails therefore to be logically true. On the other hand, we are precluded from mapping 'and' to 'or' and vice versa, unless we also map negation to itself and the True to the False. This is because on this proposal, there are two kinds of permutations, those that switch truth values and those that do not. The latter must be the identity permutation on the connectives mapping 'and' to 'and', 'or' to 'or', and so on. Obviously on such 
permutations, $\ulcorner P$ or not- $P\urcorner$ is mapped into itself, and hence into something which is coreferential with $\pi$ (True) which is $d$ (True). On those permutations that switch values, 'and' is mapped to 'or', and vice versa, negation is mapped to itself, so that $\ulcorner P$ or not- $P\urcorner$ is mapped into $\ulcorner P$ and not- $P\urcorner$ which again is coreferential with $\pi$ (True). But then $\ulcorner P$ or not- $P\urcorner$ turns out to be a logical truth after all (and similarly for all truth-functional and quantificational truths).

Under this proposal, many more propositions will appear to be logically true than under ordinary first-order logical validity. For instance, insofar as numbers are themselves courses-of-values, arithmetical propositions turn out to be logically true. But also many propositions of set-theoretic nature may turn out to have this character, as well as many that we would nowadays regard with suspicion. ${ }^{33}$ For instance, the existence of two distinct courses-of-values would turn out to be a logical truth, while the existence of objects that are not courses-of-values appears not to be (under the assumption that there are infinitely many courses-of-values and that $a$ does not denote a course-of-values, arrange for $\pi$ to be a permutation such that both $\pi(a)$ and $\pi(\pi(a))$ denote courses-of-values, and $\pi(P) \pi(x)$ holds if and only if $P x$ whenever $x$ denotes a course-of-values - then the proposition that $a$ is not a course-of-values is false under $\pi$ ).

This proposal, while still preliminary, seems to us to fit in well with a Fregean outlook. First and perhaps foremost, notice the exquisitely Fregean assumption that we have an independent purchase of the notion of being a "course-of-values" and that the truth values are themselves course-of-values (in [12], §10, Frege famously emphasizes the consistency of the assumption). Second, the method we have described, with its dependence on Frege's permutation method to capture invariance, does provide within the constraints of Frege's New Science a characterization of the "logical words". It does so, however, not by providing necessary and sufficient conditions for an atomic expression to be a logical constant. Rather, it is an indirect account that implicitly characterizes the class of logical constants by first identifying a class of propositions as logically true, and then on the basis of such a characterization, a class of terms is identified as the class of logical constants. Moreover, the Fregean approach we have described proceeds impredicatively by characterizing the class of logical constants "wholesale" as a largest class of terms satisfying a certain condition. It is in this sense a holistic account of the logical terms. Note that in this regard the Fregean approach contrasts with the Tarski-Sher-McGee account which directly identifies a subclass of the vocabulary as the class of logical terms by providing necessary and sufficient conditions for each term of that vocabulary to be a logical constant; in this regard the approach is more atomistic than the Fregean approach. ${ }^{34}$ But, of course, in comparing the two approaches the main question is whether they characterize the same class of logical terms. It appears, in fact, that the Fregean account is broader; not only does it include the classical logical terms and the cardinality quantifiers, but also, as pointed out, the proposal vouchsafes for a number of propositions of mathematical character to be logical truths. It follows that a number of terms from arithmetic and set theory (plus, times, union, intersection, and so on) turn out to be logical terms, which Frege would have liked (even if we do not).

At the end of the day, a few simple facts follow concerning logical objects and logical truths. The logical objects can simply be seen to comprise the courses-ofvalues of a pure Fregean universe (truth values, numbers, sets, and so on). Then, all true propositions concerning the logical objects are logically true. But although this 
characterization can be formulated independently of the permutation argument and the extensions and variations we have been considering, it is hard to see how it can be justified independently of it. It is precisely the task of the New Science to supply such a justification. ${ }^{36}$

\section{Concluding Remark}

The account of logical constants we have just sketched is, of course, not one that Frege himself gave, or even indicated that he envisaged; nevertheless, we believe that it is an account given exclusively in terms that are not too distant from Frege's own and hence an account which is completely within the purview of Frege's New Science. In particular the account resides solely within the object language and assumes no reinterpretation of terms. With the account, however, come a number of issues of substantial interest. For instance, is this the only sort of characterization of logical constants open to Frege? (One could imagine, taking Frege's geometrical notation seriously, that the logical constants might be characterizable through their invariance under translations of the horizontal and vertical strokes in the Begriffsschrift). Perhaps an even more important issue is the extent of the New Science. What further metatheoretical results beyond independence can be obtained within the constraints of the concepts and methods of the New Science? The answer to this question, however, lays beyond what we have tried to achieve here, which is to provide an account of the logical constants that is exactly the sort of account that is needed to complete Frege's 1906 "foray into metalogic" and, therefore, to make precise his position in the dispute with Hilbert concerning the status and independence of the axioms of geometry.

\section{Notes}

Frege and Hilbert met and conversed at a conference in Lübeck in 1895 and briefly corresponded at that time. The substantive aspects of their debate were stimulated by Frege's receipt of a copy of Hilbert's lectures of 1898-99 (although not from Hilbert but loaned by Liebmann to whom he returned it in late July 1900 with a covering letter outlining his objections) and the subsequent publication of Hilbert [22].

2. See especially van Heijenoort [39], Goldfarb [17], and Ricketts [29]. For critical response to this view, see Stanley [33], Tappenden [34], Heck [19], and Section 2 below.

3. This point has been emphasized in particular by Heck in [19].

4. This is not quite correct; he made one further, rather cryptic remark. In 1912, Jourdain published "The development of the theories of mathematical logic and the principles of mathematics" [23], a substantial portion of which was devoted to Frege's work and which included as notes remarks by Frege penned in 1910. Among these is the following: "The indemonstrability of the axiom of parallels cannot be proved. If we do this apparently, we use the word 'axiom' in a sense quite different from that which is handed down to us." Some commentators have taken this comment as indicative that Frege rejected the viability not only of Hilbert's method of proving independence but also the method 
he himself suggested in 1906 (cf. Blanchette [2], Ricketts [29]). Tappenden, however, presents an extended exegesis to the contrary arguing, effectively in our opinion, that Frege's remark is plausibly understood only as directed against Hilbert's method and not against his own (see Tappenden [35]).

5. The universality of language resides with the senses that can be expressed; they do not vary from language to language and are equally expressible in any language. In contrast, the symbols are not universal but are an arbitrary characteristic of particular languages. Typically, languages differ because they incorporate different symbols; but note that we would also have distinct languages if the symbols as well as the senses were invariant but the mappings between them were different. This would give distinct sets of propositions. This aspect of Frege's view of language, we shall see, is central to his critique of Hilbert.

6. To be a bit more precise, reasoning is carried out in a logistic system in which certain propositions formulable in the language are designated as axioms, and other propositions follow from them, via the rules of inference, as theorems. Insofar as a language is "logically perfect" we can construct logistic systems in which this method of reasoning can be reliably carried out. Not all languages measure up to this standard, most notoriously, natural languages. One place they deviate is that they will have distinct signs that differ in only one of their coordinates; in natural language we have both synonymy (difference of symbol, sameness of sense) and homophony (sameness of symbol, difference of sense).

7. This is a view shared by Russell. Thus the latter circumstance is what Russell calls in [30] as reference being "linguistic through the phrase" which he contrasts to denoting, a matter of logical determination; it is the point of the "Grey's Elegy" argument to show that this latter notion, while not uninteresting as is the former, is nevertheless flawed.

8. Of course, in 1899 Hilbert could only claim that the criterion provides a sufficient condition for $A$ to be independent of $T$; that the criterion is also necessary had to wait for Gödel's 1930 completeness theorem. Hilbert's criterion is reminiscent of the roughly contemporary undefinability criterion proposed by Padoa in 1902: to show that a concept $C$ is not explicitly definable in terms of a set $\mathbb{C}$ of concepts over a theory $T$ it suffices to exhibit two distinct interpretations of $T$ that agree on the concepts in $\mathbb{S}$ but differ as to the interpretation of $C$. Padoa's criterion was later extended by Tarski in [37] and was shown necessary by Beth [1] in his famous definability theorem.

9. Having said this, Frege registers his scepticism, proffering the opinion that by the method "the mutual independence of the axioms of Euclidian geometry cannot be proved." Cf. discussion in endnote 4.

10. [14], p. 52. Although these remarks were explicitly directed toward Korselt, Frege clearly saw him largely as Hilbert's henchman, although he does express uncertainty as to the correspondence of Korselt's presentation to Hilbert's views.

11. Treating terms in the two ways Frege mentions, effectively as constants and variables, are the only options he permits; as Demopoulos observes in [5], Fregean languages are, as he puts it, "non-indexical" in that they contain no elements that could have variable meanings (references) other than variables. What Frege did not conceive of were hybrid nonlogical constants that have their meanings specified relative to a model structure; yet this is, in modern terms, the most natural reading of Hilbert's usage. In common with Demopoulos, we trace the impossibility of nonlogical constants under Frege's conception, 
that is, the impossibility of reinterpretation, back to implications of the doctrine of sense and reference.

12. Similar remarks obtain for Frege's treatment of consistency; what can be shown is only the consistency of the complex second-level predicate corresponding to the conjunction of Hilbert's axioms, by exhibiting a set of first-level concepts which fall within it, conveniently provided by the concepts of Euclidian geometry. This will not, however, show the consistency of the axioms of Euclidian geometry, for as with independence, nothing is shown about real propositions. It is worth pointing out that beginning in 1905, Hilbert comes up with an alternative approach to consistency proofs (see [20]). Here for the first time Hilbert considers mathematical proof itself to be a mathematical object. This approach is fully developed in Hilbert [21]. In that paper, Hilbert considers a formal system with its own syntax, now understood to comprise inference rules as well as formation rules. Hilbert then proceeds to give a consistency proof by showing that certain "propositions," for example, ' $0=1$ ' are not derivable in the system. Frege would not necessarily deny the coherence of the system in this sense and presumably that results could be derived about the system's combinatorics. The combinatorial import of Hilbert's theorem is that certain strings of symbols cannot be generated by the syntactic rules of the system. Hilbert saw this result as mathematically significant because for him this constituted a consistency proof. But to Frege its significance could only be merely formal, for all that would have been done is attribute to sentences a property of propositions. It is propositions, not their formal guises, that are consistent or not, and this is not something that requires proof; if all thoughts of a set are true, they must be consistent. With axioms this is just given, for they could not all be axioms in the first place if they were not all true. In his first letter to Hilbert on this matter in 1899, Frege makes this plain: "from the truth of axioms it follows that they do not contradict one another. There is therefore no need for a further proof."

13. Whereas the issue of what qualifies a proposition as a logical axiom is a vexed one for Frege, minimally it is only logical truths (that are self-evidently so) that can attain this status. Of course, other considerations often play a role as well, especially economy, perspicuity, applicability, and so on. But compare with the discussion at the end of the paper regarding logical truth.

14. Of the terms of the language, some will be primitive and others will be defined in terms of the primitive, although since definition is explicit, the latter terms are dispensable with regard to the class of propositions that can be expressed in the language. They may be indispensable, however, in terms of what may be proven and it is toward this goalof revealing what thoughts follow from others-that we decide which terms to treat as primitive and which to treat as defined. For example, if "number" is defined, then, Frege argues, the truths of arithmetic follow from logic, something we would miss if it were primitive.

15. For Frege, propositions are "about" the references determined by the senses of the constituent signs. This notion depends on the Fregean assumption that senses have structure, and that, in particular, complex senses such as thoughts are obtained compositionally by putting together less complex senses, in ways that roughly parallel the way in which names and predicates are put together to obtain propositions. (Cf. Frege's remarks in the opening paragraph of [15].) An arithmetic proposition can thus be characterized as a proposition all of whose object-names refer to numbers; thus, both 'Nine is the sum of four and five' and 'Nine numbers the planets' are propositions "about" numbers, but only the former is an arithmetic proposition in the requisite sense. Arithmetic propositions 
are a species of propositions all of whose object-names refer to logical objects, that is, given Frege's view in [12], that refer to courses-of-values. Note that this property of propositions is discernable independently of whether the proposition is provable (or even true) so long as we have access to the thought it expresses, and hence to whether the senses of the object-names determine courses-of-values as references.

16. One might ask whether there could be axioms of an application that are themselves justified in the "logical" way (for instance, by being appropriately self-evident) allowing that there would be logical truths proven strictly within the application. Frege presumably would have answered in the negative, for if there were such logical truths, the core logic itself would be incomplete, contrary to what Frege believed. His view thus would be that no application has axioms that are logical truths (beyond those of the core logic upon which all applications are built).

17. See also the references in note 2 .

18. See [6], p. 45. Dreben and van Heijenoort specifically mention completeness, but the intended aim of their remarks is metatheoretical results in general.

19. See [17], p. 352. Ricketts remarks similarly that "the notion of a logical schema that admits of multiple interpretations is foreign to Frege's thought." See Ricketts [28], p. 76.

20. We understand logical truths to be "about" the logical furniture (that is, logical objects) in the sense described in note 15 .

21. In these post-Tarskian days, it is common to ground the distinction of the object language and the metalanguage on the necessity to avoid the semantic paradoxes, and in particular the Liar. Tarski's results are taken to show that any language that is "semantically closed" and that therefore contains its own truth predicate is inconsistent. Then, the question naturally arises of whether an externalist conception of metatheory such as we are attributing to Frege would also run afoul of the semantic paradoxes. Indeed, Frege's Begriffsschrift is designed to be as powerful and expressive as possible, and to contain semantic notions such as truth and falsity (in fact, the Begriffsschrift contains terms denoting the True and the False, and therefore a predicate of the form ' $x=$ True'). From this point of view, the situation does not seem promising for Frege. But as Gupta has shown in [18], in order for Tarski's theorem to apply, it is necessary that the language, besides containing its own truth predicate, also has an underlying classical logic, and be capable of fine enough syntactic distinctions. If either one of the last two conditions fails, Tarski's theorem no longer applies and semantically closed languages are possible. Obviously, the first escape route through nonclassical logic was not available to Frege, but also the second one would seem to be precluded as long as Begriffsschrift contains enough arithmetical machinery to code its own syntax.

22. The requirement that the list be one to one is meant to ensure the preservation of identity. Of course, it is open to consider any mapping whatsoever, but at the cost of giving up identity as a logical notion. Cf. discussion in note 25 .

23. This point is emphasized in [34].

24. Tarski's paper "What are logical notions?" [38] is a sequel to his 1956 paper "On the concept of logical consequence" [36] in which he observes that his definition of logical 
consequence depends upon a "division of all terms of the language discussed into logical and extra-logical," but that "no objective grounds are known to me which permit us to draw a sharp boundary between the two groups of terms" (pp. 418-19). This lacuna is what is rectified in the paper of five decades later.

25. See Sher [32] and McGee [27]. Also see Feferman [9] for a critical response. Our description follows McGee. Feferman's approach differs from the approach of Tarski, Sher, and McGee in that he is interested in comparing notions across different domains, so he considers arbitrary mappings from a domain $D$ onto a domain $D^{\prime}$; the price for this added generality is that the identity relation no longer turns out to have a logical character.

26. In [36], Tarski initially canvasses a characterization of logical consequence that is very similar to Frege's characterization of independence in that it is stated in terms of permutation of the nonlogical terms of the language (cf. Tarski's condition $(F)$ ). According to Tarski, this captures the intuition that "The consequence relation cannot be affected by replacing the designations of the objects referred to . . by the designations of any other objects" (p. 415). Tarski rejects this approach in favor of the model-theoretic on the basis of one argument: that languages are insufficiently expressive to carry through the characterization. "The condition $(F)$ could be regarded as sufficient for the sentence $X$ to follow from the class $K$ only if the designations of all possible objects occurred in the language in question" (p. 416). It is unclear that Frege would be moved by such a criticism, since for him this would amount to the claim that there are objects for which there are no determining senses. An object for Frege is something that can be a reference (Bedeutung) but nothing can be a reference without a sense that determines it as such. Since every sense is in principle graspable, and hence can be expressed by a sign in the Begriffsschrift, language is fully expressive relative to the universe of objects.

27. In his writings, Frege makes no specific mention of the Erlangen program, although as Jamie Tappenden informs us, at the time of his interaction with Hilbert, Frege was almost certainly familiar with it. This is witnessed by his having taken notes on Lie and Scheffers' [25] which discusses the program. (The existence of the notes is attested in the Scholz catalogue of Frege's Nachlass; cf. entry 94.) Whether Frege had knowledge prior to this we can only speculate, although in Tappenden's estimation it is likely that he did. Tappenden remarks: "It is hard to say how much Frege would have known about the specific 1872 lecture Klein gave to inaugurate his professorship at Erlangen-that specific lecture only became known later. But it is pretty likely that Frege would have known about the general program of investigating geometries in terms of invariants as early as graduate school. Klein and Lie were around Göttingen then working with Clebsch and Voss (two of Frege's teachers) and then later Klein, then Lie, took the professorship at Leipzig, which was the big university in whose orbit Jena revolved. Frege gave a couple of lectures to the Jena Mathematical society on invariants in the early 70s" (Personal communication). But, as Tappenden emphasizes, regardless of Frege's exact familiarity with the Erlangen program, the underlying idea of permutation invanance would have been within the conceptual grasp of a working geometer of the time, as indeed Frege was; see Tappenden's discussion of the principle of duality in projective geometry in [34], pp. 245-48. (Thanks here to Jamie Tappenden.)

28. Our assumption here is that the language has a specified syntax, and that permutations respect the categorization of terms so specified. On pain of circularity, this categorization must be made independently of the concepts that are characterized with respect to the permutations; thus there is a call for criteria for placing terms of the language in one category or another (cf. Dummett [7], Chapter 4) . This call, however, may be spurious; 
such is the lesson of the most detailed and extensive discussion of seeking criteria for linguistic categorization, the debate in the late 1950s over "discovery procedures" in structural linguistics that led to the ascendancy of generative grammar in which no such criteria are assumed. (Cf. Chomsky's discussion in the Introduction to [4], especially the discussion of "evaluating properties of a completed solution" on pp. 30-33.) Notice that it might be thought that in a logically perfect language this issue would not arise, for its syntax would encode the significant categorial distinctions, in particular between proper names and quantifier terms, but that it would in languages that deviate from this standard, in particular natural languages. For these we would need criteria, and as these are applied to a particular language, we would obtain a characterization of the logical terms for that language. We would thus have only a relativized notion of logical terms, for example, the logical terms of English, and would fall short of one that applies to language in general, and hence short of a general notion of logical term. This argument, however, depends upon how natural languages are taken to depart from logical perfection. Thus, it is standardly assumed in theoretical linguistics that in natural languages proper names and quantifier terms are only superficially syntactically nondistinct, but at the appropriate level of syntactic description are distinct in a way that mirrors their distinction in the logically perfect language. (Cf. May [26], Chapter 1). (We would like to thank Bill Demopoulos and Bob Hale for discussion of these issues.)

29. Ideally, one would want $C$ to be the largest class of basic terms such that every proposition in that vocabulary, if true, is logically true. For there to be such a unique class it is sufficient that if both $C$ and $C^{\prime}$ have the property that every proposition in their vocabulary is logically true if true, then the same holds for the union of $C$ and $C^{\prime}$. This is indeed a very natural condition, and one that is obvious in the case of Tarski's permutation approach. In the case of the account of logical truths sketched above, it is not as clear that the condition holds-however plausible it might be that it does—and so we must be content with the characterization of $a$ largest set of terms satisfying the condition as a good candidate for the set of logical constants.

30. As Dummett points out in [8], it is possible to accept a more limited result since Frege's strategy of presupposing an antecedently given set of invariant terms in and of itself is not objectionable, so long as we recognize the relativity of the proof to the stipulated terms. But this is hardly satisfying; what is wanted is a general procedure by which logical terms can be identified, for only then would we have a (nonrelativized) proof of independence.

31. In effect we are only assuming the right-to-left direction of Basic Law V, what Frege referred to as Basic Law Va. Note that we do not rely upon Basic Law V to specify which concepts have courses-of-values (cf. discussion in note 36 ).

32. On propositions being "about" logical objects, see note 15 .

33. See note 36 .

34. This Fregean approach has similarities with Carnap's later theory of logical constants; in [3], Carnap characterizes logical constants precisely as the class of terms such that every sentence in that vocabulary is either valid or contra-valid. But for Carnap again the direction of the explanation is the same as in the Tarski-Sher-McGee approach: logical truths are identified by their vocabulary, and the vocabulary, in turn, is identified by the above condition. 
35. As Tarski points out in [38], pp. 151-53, on this proposal mathematical notions such as union, intersection, and so on may turn out to have logical character depending upon the interpretation of the membership relation. If membership is interpreted as predication, for example, as in Russell and Whitehead [31], then indeed it is logical. If membership is taken to be a relation between objects of the domain, as in Zermelo-Fraenkel set theory, then it is not logical, as there are only four logical relations among individual objects (the universal relation, the empty relation, identity, and diversity) and membership is not among them.

36. This realization of Frege's logicist program might strike some as an illustration of the advantages of theft over honest labor. Pages and pages of toil in [12] are now replaced by a quick semantic argument; the issues of the logical and epistemological status of Frege's principles in the derivation of arithmetic in [12] are completely bypassed. We are not immune to such a worry but we should also point out that on the proposed account exactly which notions turn out to have logical character depends on what concepts have value ranges. Of course, under Frege's Basic Law V, all of them do, but the failure of Basic Law V leaves open the question of which concepts, if any, do have a course-ofvalues. In the discussion above, we assume Frege's position in [11] that numbers are value ranges of particular concepts: on this assumption, arithmetical truths are logical truths and arithmetical notions are logical notions. However, the assumption needs to be independently justified. (Thanks to Crispin Wright and Curtis Franks for discussion of this point.)

\section{References}

[1] Beth, E. W., "On Padoa's method in the theory of definition," Nederlandse Akademie van Wetenschappen Proceedings Series A, vol. 15 (1953), pp. 330-39. MR 15,385e. 263

[2] Blanchette, P., "Frege's metatheory and the 1906 independence-test," University of Notre Dame, manuscript, 2000.

[3] Carnap, R., The Logical Syntax of Language, Humanities, New York, 1937. 267

[4] Chomsky, N., The Logical Structure of Linguistic Theory, Plenum Press, New York, 1975. 267

[5] Demopoulos, W., "Frege, Hilbert, and the conceptual structure of model theory," History and Philosophy of Logic, vol. 15 (1994), pp. 211-25. MR 95i:03003. 263

[6] Dreben, B. and J. van Heijenoort, "Introductory note to Gödel 1929, 1930, 1930a," pp. 44-59 in Kurt Gödel: Collected Works, edited by S. Feferman, Oxford University Press, New York, 1986. 253, 265

[7] Dummett, M., Frege: Philosophy of Language, Duckworth, London, 1973. 266

[8] Dummett, M., "Frege on the consistency of mathematical theories," pp. 1-16 in Frege and Other Philosophers, Clarendon Press, Oxford, 1991. 267

[9] Feferman, S., "Logic, logics, and logicism," Notre Dame Journal of Formal Logic, vol. 40 (1999), pp. 31-54. Special issue in honor and memory of George S. Boolos (Notre Dame IN, 1998). MR 1811 202. 266

[10] Frege, G., Begriffsschrift, eine der arithmetischen nachgebildete Fremdsprache des reinen Denkens, L. Nebert, Halle, 1879. 
[11] Frege, G., Die Grundlagen der Arithmetik. Eine logisch-mathematische Untersuchung über den Begriff der Zahl, W. Koebner, Breslau, 1884. Unveränderter Neudruck: M \& H. Marcus, Breslau, 1934. 268

[12] Frege, G., Grundgesetze der Arithmetik, H. Pohle, Jena, 1893, 1903. 243, 246, 252, 255, 261, 265, 268

[13] Frege, G., “Über die Grundlagen der Geometrie,” Jahresbericht der Deutschen Mathematiker Vereinigung, vol. 12 (1903), pp. 319-24, 368-75. English translation by E-H. W. Kluge in Gottlob Frege, On the Foundations of Geometry and Formal Theories of Arithmetic, Yale University Press, New Haven, 1971.

[14] Frege, G., "Über die Grundlagen der Geometrie," Jahresbericht der Deutschen Mathematiker Vereinigung, vol. 15 (1906), pp. 293-309, 377-403, 423-30. English translation by E-H. W. Kluge in Gottlob Frege, On the Foundations of Geometry and Formal Theories of Arithmetic, Yale University Press, New Haven, 1971. 243, 248, 249, 250, 251, 256, 257, 258, 263

[15] Frege, G., "Compound thoughts," pp. 55-77 in Logical Investigations, Basil Blackwell, Oxford, 1977. Translated by P. Geach (editor) and R. Stoothoff.

[16] Frege, G., Logical Investigations, Basil Blackwell, Oxford, 1977. Translated by P. Geach (editor) and R. Stoothoff.

[17] Goldfarb, W. D., "Logic in the twenties: The nature of the quantifier," The Journal of Symbolic Logic, vol. 44 (1979), pp. 351-68. MR 80j:03003. 254, 262, 265

[18] Gupta, A., “Truth and paradox," Journal of Philosophical Logic, vol. 11 (1982), pp. 1-60. MR 84i:03020a. 265

[19] Heck, R., “Frege and semantics,” Harvard University, manuscript, 1999. 262

[20] Hilbert, D., "Über die Grundlagen der Logik und der Arithmetik," in Verhandlungen des Dritten Internationalen Mathematiker-Kongresses, Leipzig, 1905. English translation in From Frege to Gödel: A Source Book in Mathematical Logic, edited by J. van Heijenoort, Harvard University Press, Cambridge, 1967, pp. 129-38.

[21] Hilbert, D., "Neubegrundung der Mathematik: Erste Mitteilung," in Abhandlungen aus dem Mathematischen Seminar der Hamburgiuschen Universität 1, 1922. English translation in From Brouwer to Hilbert, edited by P. Mancosu, Oxford University Press, Oxford, 1998, pp. 198-214. 264

[22] Hilbert, D., Foundations of Geometry, Open Court, La Salle, 1971. English translation by Leo Unger. 247, 249, 262

[23] Jourdain, P., "The development of the theories of mathematical logic and the principles of mathematics," The Quarterly Journal of Pure and Applied Mathematics, vol. 43 (1912), pp. 237-69. 262

[24] Korselt, A., “Über die Grundlagen der Geometrie," Jahresbericht der Deutschen Mathematiker Vereinigung, vol. 12 (1903), pp. 402-7. English translation by E-H. W. Kluge in Gottlob Frege, On the Foundations of Geometry and Formal Theories of Arithmetic, Yale University Press, New Haven, 1971.

[25] Lie, S., and G. Scheffers, Geometrie der Berührungstransformationen, BG Teubner, Leipzig, 1896. 266

[26] May, R., Logical Form: Its Structure and Derivation, The MIT Press, Cambridge, 1985. Reprinted as "Logical Form as a Level of Linguistic Representation" in Readings in the Philosophy of Language, edited by P. Ludlow, The MIT Press, Cambridge, 1997. 
[27] McGee, V., “Logical operations," Journal of Philosophical Logic, vol. 25 (1996), pp. 56780. MR 97m:03010. 266

[28] Ricketts, T., "Objectivity and objecthood: Frege's metaphysics of judgment," pp. 65-95 in Frege Synthesized: Essays on the Philosophical and Foundational work of Gottlob Frege, edited by L. Haaparantaand and J. Hintikka, D. Reidel, Dordrecht, 1986. 265

[29] Ricketts, T., "Frege's 1906 foray into metalogic," Philosophical Topics, vol. 25 (1997), pp. 169-88. 262, 263

[30] Russell, B., “On denoting,” Mind, vol. 14 (1905), pp. 479-93. 263

[31] Russell, B., and A. N. Whitehead, Principia Mathematica. 3 volumes, Cambridge University Press, Cambridge, 1910 and 1912 and 1913. 2d edition, 1925 (Vol. 1), 1927 (Vols 2, 3). 268

[32] Sher, G., The Bounds of Logic. A Generalized Viewpoint, The MIT Press, Cambridge, 1991. MR 94g:03014. 266

[33] Stanley, J., “Truth and metatheory in Frege," Pacific Philosophical Quarterly, vol. 77 (1996), pp. 45-70.

[34] Tappenden, J., "Metatheory of mathematical practice in Frege," Philosophical Topics, vol. 25 (1997), pp. 213-63. 253, 262, 265, 266

[35] Tappenden, J., "Frege on axioms and independence arguments in geometry: Did Frege reject independence arguments?" University of Michigan, manuscript, 2000. 263

[36] Tarski, A., "On the concept of logical consequence," pp. 409-20 in Logic, Semantics, Metamathematics, edited by J. Corcoran, Clarendon Press, Oxford, 1956. Translated by J. H. Woodger. 265, 266

[37] Tarski, A., "Some methodological investigations on the definability of concepts," pp. 296319 in Logic, Semantics, Metamathematics, edited by J. Corcoran, Clarendon Press, Oxford, 1956. Translated by J. H. Woodger. 263

[38] Tarski, A., "What are logical notions?" History and Philosophy of Logic, vol. 7 (1986), pp. 143-54. MR 88b:03010. 265, 268

[39] van Heijenoort, J., "Logic as calculus and logic as language," Synthese, vol. 17 (1967), pp. 324-30. 262

\section{Acknowledgments}

We are indebted to Jamie Tappenden for very helpful discussions and for sharing with us some of his unpublished work (cf. references above), and to Tim Williamson for providing valuable comments on a previous draft of the paper.

Department of Logic and Philosophy of Science

School of Social Sciences

University of California Irvine

Irvine CA 92697-5100

aldo@uci.edu

http://kleene.ss.uci.edu/ aldo

rmay@uci.edu

http://kleene.ss.uci.edu/ rmay 